\title{
Face Verification Based on Bagging RBF Networks
}

\author{
Yunhong Wang ${ }^{1}$, Yiding Wang ${ }^{2}$, Anil K. Jain ${ }^{3}$, and Tieniu Tan ${ }^{4}$ \\ ${ }^{1}$ School of Computer Science and Engineering, Beihang University, Beijing, 100083, China \\ yhwang@buaa.edu.cn \\ ${ }^{2}$ Graduate School, Chinese Academy of Sciences, Beijing, 100049, China \\ ydwang@gscas.ac.cn \\ ${ }^{3}$ Department of Computer Science \& Engineering, \\ Michigan State University, East Lansing, MI 48824 \\ jainacse.msu.edu \\ ${ }^{4}$ National Laboratory of Pattern Recognition, Institute of Automation, Chinese Academy of \\ Sciences, P.O. Box 2728, Beijing 100080, P.R. China \\ tntenlpr.ia.ac.cn
}

\begin{abstract}
Face verification is useful in a variety of applications. A face verification system is vulnerable not only to variations in ambient lighting, facial expression and facial pose, but also to the effect of small sample size during the training phase. In this paper, we propose an approach to face verification based on Radial Basis Function (RBF) networks and bagging. The technique seeks to offset the effect of using a small sample size during the training phase. The RBF networks are trained using all available positive samples of a subject and a few randomly selected negative samples. Bagging is then applied to the outputs of these RBF-based classifiers. Theoretical analysis and experimental results show the validity of the proposed approach.
\end{abstract}

\section{Introduction}

Systems based on face recognition and verification play an important role in applications such as access control, credit card authentication, video surveillance, etc., where the identity of a user has to be either determined or validated. Although face recognition and face verification use similar algorithms [1], they are two different problems with different inherent complexities [2]. Recognition is an $\mathrm{N}$-class problem, where the input face image is mapped to one of the $\mathrm{N}$ possible identities, whereas verification is a 2-class problem, where the input image is mapped to one of two classes, genuine or impostor. In other words, recognition necessitates a one-to-many matching, while verification requires a one-to-one matching. In designing a classifier for face verification, both positive and negative learning samples are needed. Usually, a very small number of positive (genuine) samples and a very large number of negative (impostor) samples are available during training. Thus, the classifier will be over-fitting the impostor samples while it is learning using only a few positive samples. Simply put, the generalization ability of the classifier during the training stage is very low. This could be one reason why face 
verification systems do not achieve high matching accuracy. In this paper, we will introduce a technique to decrease this effect by non-equilibrium training. A radial basis function (RBF) network is a good classifier for face recognition because it has the ability to reduce misclassifications among the neighboring classes [6]. Another advantage of RBF network is that it can learn using both positive and negative samples [10]. This property motivates the choice of RBF network for face verification. We train several RBF networks for verification, and we boost the performance by bagging the results of these various networks. There are many methods for face verification described in the literature [3][4][5][10]. Most of them operate by training a classifier that is unique for each subject, although the structure of the classifier is the same for all subjects. Theoretically, the number of possible impostor samples for a subject should be much larger than the number of genuine samples. In practice, only a subset of impostor samples is used for training and, hence, the impostor space cannot be established very well. However, we cannot collect samples of all possible impostors. This makes it difficult to arrive at a reasonable estimation of the probability space of impostors. Therefore, we will not attempt to estimate the probability of impostor space by using all possible impostor samples. Rather, we use some of the samples selected randomly from the impostor database (along with all available genuine samples) in the training stage of each RBF classifier. The number of training samples for each classifier is small compared to the dimensionality of the data (number of features). Usually, a classifier that is constructed using a small training set is biased and has a large variance since the classifier parameters are poorly estimated. Consequently, such a classifier may be weak, having a poor performance [7]. Bagging is a good technique to combine weak classifiers resulting in a powerful decision rule.

In this paper, we use bagging to combine the RBF classifiers in order to improve the accuracy of face verification. The rest of the paper is organized as follows: Section 2 introduces face feature extraction and a classifier based on RBF networks and bagging; experimental results are given in Section 3; Section 4 presents a discussion and summary of the work.

\section{Face Verification}

\subsection{The Problem of Face Verification}

The verification problem can be formulated as follows. Classify a test sample $\mathrm{S}$ (a face image) into one of the following two classes: $\omega_{0}$ (genuine) or $\omega_{1}$ (impostor). Let $\mathrm{Y}$ be a feature vector extracted from $S$, then

$$
\begin{aligned}
& \text { Assign } \quad \mathrm{S} \rightarrow \omega_{j} \quad \text { if } \\
& \qquad P\left(\omega_{j} \mid Y\right)=\max _{k=0}^{1} P\left(\omega_{k} \mid Y\right) \mathrm{j}=0,1
\end{aligned}
$$

Where $P\left(w_{k} \mid Y\right)$ denotes the posteriori probability of $w_{k}$ given $\mathrm{Y}$. 


\subsection{Feature Representation Using Eigenface}

We use the eigenface technique to represent a face image [9]. Let the ith sample face image be represented as an $\mathrm{N}$-dimensional vector $X_{i}, \mathrm{i}=1,2 \ldots \mathrm{n}$. The scatter matrix $S$ of all the n samples is computed as

$$
S=\sum_{i}\left(X_{i}-\mu\right)\left(X_{i}-\mu\right)^{T}
$$

Where $\mu$ is the mean vector. Here, only a portion of the available database is used to create the eigenspace. For each image $\mathrm{X}$, we obtain a feature vector $\mathrm{Y}$ by projecting $\mathrm{X}$ onto the subspace generated by the $\mathrm{M}$ principal directions, according to the following equation:

$$
Y=W^{T} X
$$

Images are compared by finding the distance between their corresponding feature vectors. In our face verification problem, we represent each face sample as a 40-dimensional $(M=40)$ and a 10-dimensional $(M=10)$ vector, respectively. Since the first 3 eigenvectors are related to the variation in illumination (see, Pentland [9]), we eliminate the first 3 eigenvectors for every face sample. Each subject is trained using a different classifier.

\subsection{RBF Neural Network}

The output of the jth hidden node in a RBF network can be expressed as [11]:

$$
O_{h k}=\Phi\left(\left\|Y_{k}-\mathbf{C}_{j}\right\|\right) \mathrm{j}=1,2, \ldots N_{0}
$$

where $Y_{k}$ is a M-dimensional input vector, $\mathbf{C}_{j}$ is the center of the jth RBF network, $N_{0}$ is the number of hidden units, and $\Phi(\cdot)$ is a nonlinear, radial symmetric function whose center is $C_{j}$. We use the Gaussian function as the basis function. The output of the hidden layer can be written as:

$$
O_{h k}=\Phi\left(\left\|Y_{\mathbf{k}}-\mathbf{C}_{j}\right\|\right)=\exp \left[-\sum_{i=1}^{M} \frac{\left(y_{i}-C_{i j}\right)^{2}}{2 \rho_{j}^{2}}\right]
$$

The output of the ith output unit of the RBF network is:

$$
z_{k i}=\sum_{h} w_{i h} \Phi\left(\left\|Y_{k}-\mathbf{C}_{\mathbf{j}}\right\|+w_{k 0}\right.
$$

We use the training samples to compute the center, $\mathbf{C}_{j}$. The $\rho_{j}$ s are selected based on the method in [6], namely, that $\rho_{j}$ is computed based on inter-class and intra-class distances. One of the advantages of RBF network is that it can be trained using both 
positive and negative samples. Note that since we are dealing with a verification problem, we can build an individual network for each subject in the database. This is because, for verification, an unknown individual must claim his identity first, and therefore we would know which network to use.

\subsection{Bagging}

Bagging has proven to be a useful method to improve the classifier performance by combining individual classifiers. Such a combination often gives better results than any of the individual classifiers [8]. As mentioned above, RBF classifiers we have used are weak classifiers. It is necessary to boost their performance by using a bagging technique. Bagging is implemented in the following way [8]:

1. Let $b=1,2, \ldots, \mathrm{B}$ be the training samples available for a subject. The following two steps are done for each $b$ :

(a) Take a bootstrap replicate $Z^{b}$ of the training data set $\mathrm{Z}$.

(b) Construct a classifier $C^{b}(z)$ (with a decision boundary $C^{b}(z)=0$ ) on $Z^{b}$.

2. Combine classifiers $C^{b}(z), \mathrm{b}=1,2, \ldots \mathrm{B}$, by a simple majority voting (the most often predicted label) to obtain the final decision rule as follows:

$$
\beta(z)=\underset{y \in\{-1,1\}}{\arg \max } \sum_{b} \delta_{\mathrm{sgn}\left(C^{b}(z)\right), y} \text { Where } \delta_{i, j}=\left\{\begin{array}{l}
1, i=j \\
0, i \neq j
\end{array}\right.
$$

is the Kronecker symbol, $y \in\{-1,1\}$ is a decision (class label) of the classifier.

Note that there are multiple RBF networks trained for one subject so the decision is made by majority voting. The network classifiers are combined using the bagging rule. We have used different number of classifiers $(1,5,10,15$, and 20 , respectively) in our experiments. To evaluate the performance of bagging, we conduct another experiment in which only a single RBF network is used for every subject. The features are once again extracted using the eigenface technique. The negative (impostor) samples correspond to the genuine samples of all the other subjects. All the negative samples are used during training along with all the available positive samples. This method is represented as PCA+RBF in Table 2 and Table 4.

\subsection{Universal and Individual Eigenface Method}

We compare the proposed method to two existing approaches to face verification: the universal eigenface and individual eigenface methods [2]. The universal eigenface method constructs an eigenspace using all the training data available for all the subjects. The templates are the coefficients of the projected vectors in the above eigenspace. The distance between the coefficients of a test image and the template is used as a matching score. If the matching score exceeds a threshold, it is declared to be an impostor. Here we use different thresholds for each subject. The thresholds are proportional to the variability of interclass and intraclass. The basic idea of the individual eigenface method [2] is to capture the intra-class variations of each subject, such as changes in expression, illumination, age, etc. In the individual PCA approach, 
one eigenspace is constructed for each training subject. The residue of a test vector to that vector's individual eigenspace (i.e., the squared norm of the difference between a test vector and its representation in the eigenspace) is used to define the matching score. The thresholds are computed from the training set. We set different thresholds for each subject.

\section{Experimental Results}

\subsection{Database}

We use the ORL [8], Yale [12] and NLPR databases for face verification. While the first two are well known public domain face databases, NLPR consists of face images taken in national lab of pattern recognition (NLPR) at two different time instances. Examples of typical face images from NLPR databases are shown in Figure 1. The ORL database contains 40 subjects and 400 images. The Yale database contains 15 subjects and 165 images. The NLPR database contains 19 subjects and 266 images. The above databases are composed of faces with reasonable variations in expression, pose and lighting. We use 6 samples per subject as the positive (genuine) training data. All the images are preprocessed to decrease the effect of variations in illumination. To test our proposed approach on a larger database, we combine all the three databases. The integrated database includes the ORL, NLPR, Yale and MIT databases. The first three databases are introduced above. The MIT database contains 16 subjects and 432 images. There are 90 subjects in the integrated database.

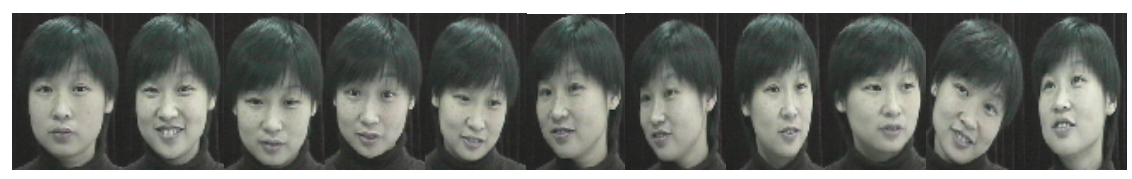

Fig. 1. NLPR face Database

\subsection{Experimental Results}

The experiments were conducted in the following way: Firstly, all the images in the training set are mapped to the eigenspace to generate the projected feature vectors. For each subject, we select 6 samples (randomly) as positive training samples and the images of the remaining subjects are regarded as impostors. Half of the samples coming from the other subjects are used as negative training data. Secondly, 6 samples are randomly selected from the negative training data and combined with the positive training data. This data is used to train several RBF classifiers. Finally, the output of these RBF classifiers are bagged using the methods described in 2.4. The results are shown in Tables 1 and 2. Table 2 gives the verification results using the universal and individual eigenface methods. The PCA+RBF method is used to refer to the technique where we first extract features of each subject via the eigenface method, and then use all the training samples to construct a RBF classifier for verification. This method has been mentioned in Section 2.4. We use 40-dimensional and 10-dimensional eigenfeatures to realize the proposed methods. 
Table 1. Verification error of bagging RBF classifiers (all the impostor subjects are used in training) on the ORL database

\begin{tabular}{ccccc}
\hline \multirow{2}{*}{$\begin{array}{c}\text { Number of } \\
\text { classifiers }\end{array}$} & \multicolumn{2}{c}{ FRR (\%) } & \multicolumn{2}{c}{ FAR (\%) } \\
\cline { 2 - 5 } & \multicolumn{2}{c}{ Dimension of features } & \multicolumn{2}{c}{ Dimension of features } \\
\hline & 40 & 10 & 40 & 10 \\
\hline 1 & 17.75 & 12.50 & 24.48 & 21.32 \\
\hline 5 & 2.50 & 2.30 & 3.87 & 3.79 \\
\hline 10 & 0 & 0 & 3.06 & 1.18 \\
\hline 15 & 0 & 0 & 4.51 & 2.56 \\
\hline 20 & 0 & 0 & 6.00 & 4.53 \\
\hline
\end{tabular}

Table 2. The Verification Error Rates on Yale and NLPR databases (10 dimensional feature space; all the impostor subjects are used in training)

\begin{tabular}{ccccc}
\hline $\begin{array}{l}\text { Number } \\
\text { classifiers }\end{array}$ & \multicolumn{2}{c}{ Yale Database } & \multicolumn{2}{c}{ NLPR database } \\
\hline & FRR & FAR & FRR & FAR \\
\hline 1 & 20.32 & 28.58 & 25.33 & 32.38 \\
\hline 5 & 10.26 & 12.63 & 16.42 & 21.84 \\
\hline 10 & 3.72 & 4.19 & 4.85 & 6.62 \\
\hline 15 & 4.27 & 6.35 & 5.62 & 8.39 \\
\hline 20 & 4.86 & 7.07 & 6.16 & 8.85 \\
\hline
\end{tabular}

It has to be noted that in an operational system, some of the impostor samples, with respect to a single subject, cannot be acquired during the training phase. We refrain from using all the impostor subjects for training and testing the performance of the verification system. Instead, we use a subset of the impostors during training. Thus, the testing database contains some subjects that are not presented in the training database. The results of bagging the RBF classifiers are shown in Tables 4 and 5. Table 6 gives the verification results of the universal and individual eigenface methods. We can see that the results with the NLPR and Yale databases are not as good as that of the ORL database. The reason is that there are more variations in the NLPR and Yale databases which affect the accuracy of face verification. This can not be compensated for by learning. The error rates of bagging on the integrated database is even lower than that

Table 3. The verification error rates of Universal eigenface (UEigenface), individual eigenface(IEigenface), PCA+RBF, and bagging , (10 dimensional feature space)

\begin{tabular}{|c|c|c|c|c|c|c|c|c|}
\hline \multirow[t]{2}{*}{ Verification System } & \multicolumn{2}{|c|}{$\begin{array}{l}\text { ORL } \\
\text { Database }\end{array}$} & \multicolumn{2}{|c|}{$\begin{array}{c}\text { Yale } \\
\text { Database }\end{array}$} & \multicolumn{2}{|c|}{$\begin{array}{l}\text { NLPR } \\
\text { Database }\end{array}$} & \multicolumn{2}{|c|}{$\begin{array}{l}\text { Integrated } \\
\text { Database }\end{array}$} \\
\hline & FRR & FAR & FRR & FAR & FRR & FAR & FRR & FAR \\
\hline UEigenface & 4.51 & 4.20 & 6.74 & 6.79 & 8.19 & 8.86 & 12.57 & 15.72 \\
\hline IEigenface & 3.30 & 2.81 & 5.41 & 4.92 & 6.63 & 6.06 & 8.15 & 7.82 \\
\hline $\mathrm{PCA}+\mathrm{RBF}$ & 5.00 & 8.74 & 14.57 & 12.59 & 18.71 & 16.62 & 23.71 & 21.80 \\
\hline Bagging RBF & 0 & 1.18 & 3.72 & 4.19 & 4.85 & 6.62 & 5.73 & 6.88 \\
\hline
\end{tabular}


Table 4. Verification Error Rates of bagging RBF classifiers on ORL database

\begin{tabular}{ccccc}
\hline \multirow{2}{*}{$\begin{array}{c}\text { Number of } \\
\text { classifiers }\end{array}$} & \multicolumn{2}{c}{ FRR (\%) } & \multicolumn{2}{c}{ FAR (\%) } \\
\cline { 2 - 5 } & \multicolumn{2}{c}{ Dimension } & \multicolumn{2}{c}{ Dimension } \\
\hline & 40 & 10 & 40 & 10 \\
\hline 1 & 19.78 & 13.55 & 24.48 & 25.65 \\
\hline 5 & 4.50 & 2.60 & 8.73 & 4.82 \\
\hline 10 & 0 & 0 & 3.12 & 1.24 \\
\hline 15 & 0 & 0 & 4.64 & 2.69 \\
\hline 20 & 0 & 0 & 6.25 & 4.62 \\
\hline
\end{tabular}

Table 5. The Verification Error Rates on Yale and NLPR databases (10 dimensional feature space, part of the impostor database is used in training)

\begin{tabular}{ccccc}
\hline $\begin{array}{c}\text { Number of } \\
\text { classifiers }\end{array}$ & \multicolumn{2}{c}{ Yale Database } & \multicolumn{2}{c}{ NLPR database } \\
\hline & FRR & FAR & FRR & FAR \\
\hline 1 & 21.57 & 27.65 & 28.39 & 34.63 \\
\hline 5 & 11.48 & 12.93 & 17.16 & 20.96 \\
\hline 10 & 3.74 & 4.31 & 5.02 & 6.52 \\
\hline 15 & 4.47 & 6.81 & 5.78 & 8.85 \\
\hline 20 & 4.92 & 7.85 & 6.42 & 9.10 \\
\hline
\end{tabular}

Table 6. The verification rates of Universal Eigenface (UEigenface), Individual Eigenface(IEigenface), PCA+RBF, and bagging, (10 dimensional feature space, part of the impostor subjects are used in training)

\begin{tabular}{lcccccccc}
\hline Verification System & \multicolumn{2}{c}{ ORL } & \multicolumn{2}{c}{ Yale } & \multicolumn{2}{c}{$\begin{array}{c}\text { NLPR } \\
\text { Database }\end{array}$} & \multicolumn{2}{c}{$\begin{array}{c}\text { Integrated } \\
\text { Database }\end{array}$} \\
& Database & \multicolumn{2}{c}{ Database } & \multicolumn{2}{c}{ Dab } & FRR & FAR \\
\hline URR & FAR & FRR & FAR & FRR & FAR & FRR \\
\hline UEigenface & 4.59 & 5.3 & 8.84 & 9.19 & 11.10 & 12.21 & 12.91 & 14.64 \\
\hline IEigenface & 3.60 & 3.48 & 6.72 & 5.53 & 8.83 & 8.80 & 9.71 & 9.10 \\
\hline PCA+ RBF & 5.00 & 9.89 & 18.16 & 19.37 & 24.31 & 28.89 & 25.92 & 26.61 \\
\hline Bagging RBF & 0 & 1.24 & 3.74 & 4.31 & 5.02 & 6.52 & 5.62 & 6.61 \\
\hline
\end{tabular}

on the NLPR. The reason is that the eigenface created by the different databases emphasizes different variations (illumination, pose etc.). The differences among the subjects are not emphasized in this way. That means that the eigenfeature of every subject is not as 'significant' as in the individual database.

\subsection{Discussions}

We have applied bagging on the outputs of multiple RBF classifiers to improve the performance of a face verification system. It has been shown that the proposed method has better matching performance than the universal eigenface and individual eigenface methods. One of the advantages of the proposed approach is the use of a subset of the subjects as impostors during training without compromising the verification 
performance. Another advantage of the proposed approach is that its verification accuracy is not proportional to the number of classifiers. Using a large number of classifiers does not result in a higher accuracy of verification. In our proposed system, 10 classifiers are sufficient for bagging. The reason for this could be that the feature vector is 10-dimensional while the number of training samples for every classifier is only 12.Experimental results show that the 10-dimensional feature vector gives better verification results than the 40-dimensional feature vector. The error rate of bagging RBF does not decrease dramatically when only a subset of the impostor samples is employed in training, while other face verification methods do not have this advantage. The error rates on Yale database and NLPR database are high because there are many variations in illumination and pose in these two databases. Considering that only 6 randomly selected samples are used in the training phase, these results are reasonably good. This is typical in real systems since often we can only get a small number of positive samples that may not be typical ones for a person.

\section{Conclusions}

In summary, the proposed approach not only has a good accuracy but also has a good generalization capability. The accuracy may be attributed to the following: (i) The RBF classifier can learn not only from positive samples but also from negative samples. (ii) We have selected the negative samples randomly and combined them with an equal number of positive samples. This will decrease the over-fitting of negative subjects. (iii) The random choice of negative samples enhances the generalization ability that is useful when all the impostor samples are not available.

\section{Acknowledgements}

We would like to thank Arun Ross for a careful reading of this paper. This research was supported by a grant from the Chinese NSFC (No.60332010).

\section{References}

1. C. L. Kotropoulos, C. L. Wilson, S. Sir Ohey, Human and Machine Recognition of Faces: a Survey, Proc. IEEE 83(5), (1995), 705-741.

2. Xiaoming Liu, Tsuhan Chen, and B. V. K. Vijaya Kumar, Face authentication for multiple subjects using eigenflow, Pattern Recognition, 36(2), (2003), 313-328,

3. Gian luca marcialis and Fabio Roli, Fusion of LDA and PCA for Face Verification, Biometric Authentication, LNCS 2359, Proc. of ECCV 2002 Workshop, (2002), 30-37.

4. http://www.ece.cmu.edu/ marlene/kumar/Biometrics_AutoID.pdf

5. Yunhong Wang, Tieniu Tan and Yong Zhu, Face Verification Based on Singular Value Decomposition and Radial Basis Function Neural Network, Proceedings of Asian Conference on Computer Vision, ACCV, (2002), 432-436.

6. Meng Joo Er, Shiqian Wu, Juwei Lu, and Hock Lye Toh, Face Recognition With Radial Basis Function (RBF) Neural Networks, IEEE Trans. on NN, 13(3), (2002), 697-710. 
7. Marina Skurichina and Robert P. W. Duin, Bagging, Boosting and the Random Subspace Method for Linear Classifiers, Pattern Analysis \& Applications, 5 (2002) 121-135,

8. Ferdinando Samaria and Andy Harter, Parameterization of a Stochastic Model for Human Face Identification, in Proc. $2^{\text {nd }}$ IEEE workshop on Applications of Computer Vision, Sarasota, FL, 1994.

9. M. Turk and A. Pentland, Eigenfaces for Recognition, Journal of Cognitive Neuroscience, 3(1), (1991), 71-86.

10. Simon Haykin, Neural Networks: A Comprehensive Foundation, MacMillan Publishing Company, 1994.

11. http://cvc.yale.edu/projects/yalefaces/yalefaces.html. 